\title{
TREATMENT OF THE ISCHAEMIC LIMB WITH HIGH PRESSURE OXYGEN
}

\author{
Captain N. K. CONI, M.A., M.R.C.P., R.A.M.C. \\ Lieutenant R. R. URRY, S.R.N., R.A.M.C. \\ Cambridge Military Hospital, Aldershot
}

DURING the last few years, considerable interest has attached to the therapeutic possibilities of hyperbaric oxygen in a variety of disorders. So far, two conditions have emerged in which this form of treatment is of undoubted benefit carbon monoxide poisoning and gas gangrene. Apart from these, the technique has been applied for the most part to situations in which the predominating feature is arterial insufficiency. This may be due to traumatic interruption of an artery or to arterial disease, degenerative or inflammatory, which is occluding or threatening to occlude its lumen. In any event, the result is impaired vascularization of an extremity or of the myocardium, brain, or other viscus. The following case is one example of such a condition.

\section{Case Report}

The patient, a 41 year old N.C.O, had had an anterior myocardial infarct one year previously: in November 1965 he was admitted to hospital with a four month history of angina of effort culminating in chest pain occuring at rest and persisting for hours at a time during the preceding two weeks. This had been associated with exertional dyspnoea, orthopnoea and paroxysms of nocturnal dyspnoea. In addition, during the preceding two days there had been severe pain in the left buttock, groin and thigh on walking a distance of 25 to 50 yards. He was noted to be somewhat hypotensive, febrile, and the jugular venous pressure became elevated shortly following his admission. The most striking findings, however, were in the left leg where the foot was cold and cyanosed with a sluggish venous return. All the leg pulses including the femoral were impalpable whereas all pulses were present in the right limb. The cardiogram showed evidence of established posterior infarction which had become superimposed on the changes of old anteroseptal infarction, and chest $\mathrm{x}$-ray demonstrated increase and subsequent return to normal of heart size. Sedimentation rate was $66 \mathrm{~mm}$ per hour on admission.

The diagnosis made was of recurrent myocardial infarction with cardiac failure and with probable embolism to the left common or external iliac artery. The decompensation was treated with the usual measures and he was given anticoagulants, as it was felt that surgical intervention was impossible at such a time. It was therefore decided to treat him with hyperbaric oxygen (which might prove beneficial both to the limb and the myocardium) and this was carried out for two weeks during which time he had two two-hour periods a day at a pressure of almost $2 \frac{1}{2}$ atmospheres. On this regime, and over the course of two or three weeks, the cardiac pain disappeared, the blood pressure rose again to normal levels, and it became possible to withdraw digitalis and diuretics without any recurrence of failure. The extremity of the left leg continued for the first ten days or so to be gravely threatened by gangrene but it remained viable and eventually lost its cyanotic hue, It is still completely pulseless but there is no longer any detecable difference in skin temperature between the two limbs, and he can now walk a distance of about 150 yards at a slow pace before he gets the pain of claudication.

\section{The Hyperbaric Oxygen Chamber}

The apparatus used was readily made available by Oxygenaire Limited, a part of the Medical Division of Vickers Limited, and was described by Hopkinson and Williams in 1963. Basically, it consists of an Emergency Vehicle containing a cylindrical chamber of double perspex into which a special stretcher can be introduced on runners. It is equipped with an aircraft intercommunication system to enable the nurse to converse with the patient at all times. Oxygen can be introduced under pressure through various tubes entering the chamber from four 420 cubic ft cylinders encased under the vehicle. The control panel embodies the controls and gauges required for the mains electricity, regulation of the oxygen, pressure selection, pressurising of the chamber, temperature and humidity and carbon dioxide concentration in the chamber. It also has an emergency alarm system which sounds if any part of the sequence is carried out in the wrong 


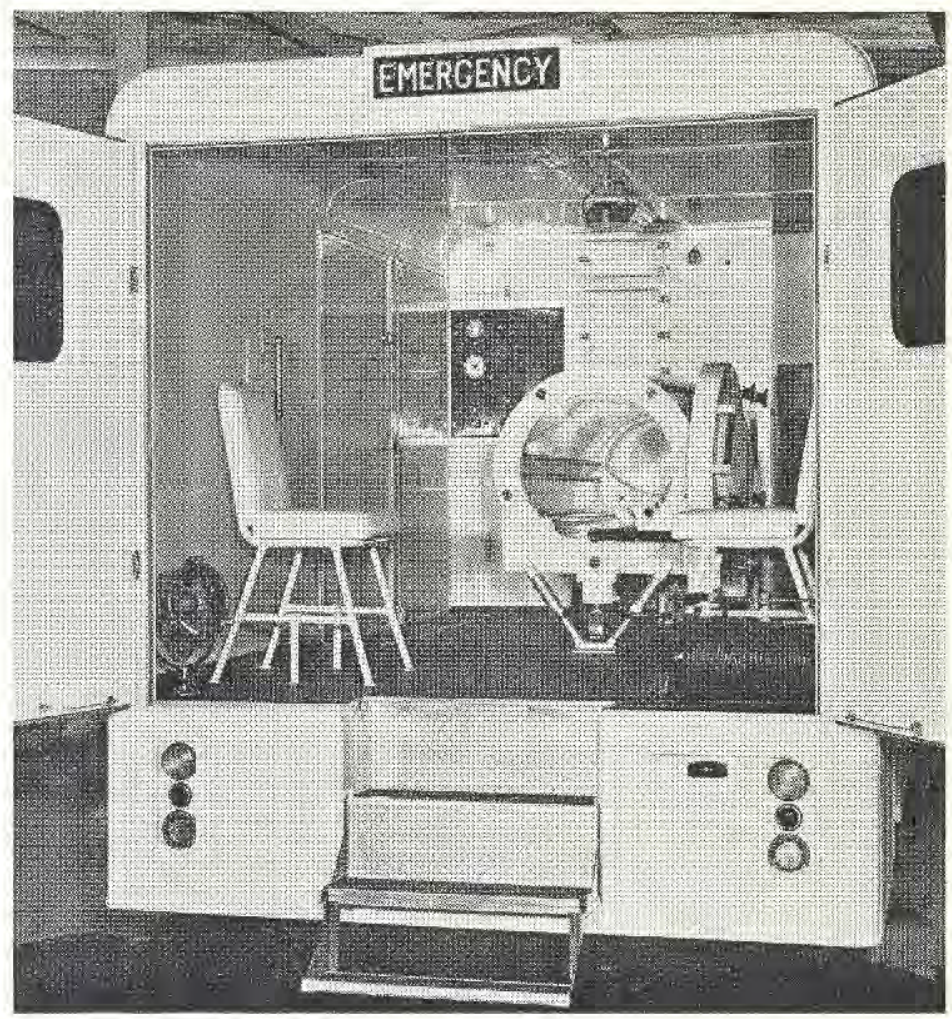

Fig. 1 The Emergency Vehicle with its one-man hyperbaric oxygen chamber

order or if the oxygen fails. Fitted to the chamber door is an automatic switch which is activated by the door being closed and flushes the chamber with oxygen. Before use the machine is connected to the mains and the oxygen cylinders are filled through a nonreturn valve situated near the cab on the outside of the vehicle. The oxygen driers are refilled with calcium carbonate, the $\mathrm{CO}_{2}$ filters are refilled with soda lime and the dewpoint reservoir under the chamber is drained. The door of the chamber is then opened and the oxygen is turned on fully. After a few minutes the mains switch and the intercommunication switch are turned on. The chamber is then ready for the patient.

The process is explained to the patient and he is made comfortable on the stretcher which is then put into the chamber and the door closed. The operator talks to the patient as he works. As the door closes the chamber is flushed with oxygen and the pressure selected, 20 Ibs per square inch (above atmospheric) being that chosen in the present instance. Compression is now started, and it is advisable to carry this out slowly on the first few occasions until the subject becomes used to it: thereafter compression and decompression can each be completed in about ten to fifteen minutes. The rate of compression that is tolerated with comfort varies greatly from one patient to another, but in general does not exceed six pounds per square inch per minute. 
If the subject complains of difficulty in hearing or of popping of the ear drums, compression is halted and he is told to chew or turn his head on one side until the sensation passes off. Pressurisation is then continued until reaching the required level which is maintained for the desired period. Decompression can be carried out more rapidly without discomfort, an average rate being five pounds per square inch per minute, and in an emergency can be accomplished so quickly that the patient is removed from the chamber in less than half a minute.

From the nursing point of view this was a new and most interesting duty to perform.

\section{Discussion}

There are available several alternative forms of management of obliterative arterial disease in a limb (anti-coagulation, fibrinolytic enzymes, surgery) but one of the most important things to be said in favour of high pressure oxygen is that it can do no harm (unless a pressure of three atmospheres is exceeded). Secondly, it is at least based on a sound theoretical rationale Under normal conditions the oxygen content of arterial blood is about 19.5 volumes per cent, of which only about a quarter of a volume is in simple solution in the plasma. By increasing the oxygen pressure it is not possible materially to increase the amount of oxygen carried in combination with haemoglobin as this is almost maximally saturated anyway: but it is possible to effect a substantial increase in this normally negligible quantity of oxygen carried in solution in the plasma. In oxygen at two atmospheres, for instance, the figure is 4.2 volumes per cent (Illingworth 1962. Bird and Telfer 1965) and this raises the total oxygen content of the blood to almost 25 volumes per cent. In the event of sudden occlusion of the main artery to a limb, such an increase might make all the difference between viability and gangrene. Either the obstruction may be incomplete, permitting a little blood to trickle past it: or else there are small anastomatic bypasses such as the gluteal arteries in the case of the lower limb which offer an alternative channel. The quantity of blood perfusing the limb will be much reduced, but as Boerema (1964) suggests, it is possible that such an increase in the oxygen which is transported by it might be sufficient to maintain the life of the tissues temporarily. This will buy valuable time during the intervening period while a collateral circulation is developing or a thrombus is undergoing recanalisation, or while the patient is being prepared for surgery.

Unfortunately, work in this field is impeded primarily by the great difficulty in determining whether the treatment is in fact producing any benefit or not, as ischaemic disorders of this type do not readily lend themselves to precise statistical evaluation. It would be virtually impossible to carry out a properly controlled trial, and recorded results therefore take the form of collections of individual cases. What does emerge is that in the case of chronic obliterative vascular disease the use of this technique probably does not affect the long-term outlook (Illingworth 1965: Ledingham 1963: Smith 1964). The picture is very much more encouraging, however, when considering the results in traumatic ischaemia: assuming the present case to be embolic it is more physiologically analogous to traumatic ischaemia than to chronic arterial disease, and in any case it is in the treatment of trauma that high pressure oxygen is likely to find its widest application in a military context. Several authors describe their experience with such cases in which there is a strong impression that real benefit has resulted from hyperbaric oxygen (Ledingham 1963: Maudsley, Hopkinson and Williams 1963: Smith, Stevens, Griffiths and Ledingham 1961: Smith 1962. Illingworth 1965: Smith 1964). 


\section{Summary}

The case is described of a patient who was thought to have an embolus in the common or external iliac artery complicating a myocardial infarct, and who was treated with hyperbaric oxygen. The object of describing this case is not to claim any striking therapeutic achievement but to present a situation in which definitive surgical treatment was considered too hazardous but the patient has nevertheless done better than anticipated with a more conservative approach. The value of high pressure oxygen in the management of the ischaemic extremity is disucssed, and it is concluded that it may well be of real value especially in the treatment of sudden and surgically reversible arterial interruption, particularly of traumatic origin.

\section{REFERENCES}

BIRD, A. D. and TELFER, A B. M. (1965). Effect of Hyperbaric Oxygen on Limb Circulation. Lancet.1, 355. BoEREMA, I. (1964). High Tension Oxygen Therapy Proceedings of the Royal Society of Medicine. $57,817$. Hopkinson, W. I. and WILLIAMs, K. G. (1963). High Pressure Oxygen Therapy.World Medical Electronics. 2,66 .

ILLINGWORTH, C. (1962). Treatment of Arterial Occlusion under Oxygen at Two-Atmospheres Pressure. $B M J 2,1271$.

ILLINGWORTH, C. (1965). Arterial Insufficiency and Hyperbaric Oxygenation Annals of the New York Academy of Science. 117, 671.

LEdingham, I. MCA. (1963). Some Clinical and Experimental Applications of High Pressure Oxygen. Proceedings of the R.S.M.56, 999.

MAUDSley, R. H., Hopkinson, W. I. and Williams, K. G. (1963). Vascular Injury Treated with High Pressure Oxygen in a Mobile Chamber. Journal of Bone and Joint Surgery, 45B, 346.

SMith, G., STEVENS, J., GRiffirhS, J. C. and LedinghaM, I. MCA. (1961). Near Avulsion of Foat treated by Replacement and Subsequent Prolonged Exposure of Patient to Oxygen at 2 Atmospheres Pressure. Lancet. 2, 1122 .

SMith, G. (1962). Traumatic Ischaemia. Postgraduate Medical Journal 38, 695.

SmITH, G. (1964). The Present Position of Hyperbaric Oxygen Therapy. Proceedings of the R.S.M. 57, 818.

\section{Honorary Consultant to the Army}

Mr. H. R. Thompson, M.A., M.B., F.R.C.S., has been appointed Honorary Consultant in Proctology to the Army as successor to Sir Clifford Naunton Morgan who retired on 20th December, 1966.

Mr. Thompson who is surgeon to St. Mark's Hospital, served with the R.A.M.C. during the second world war in North West Europe and India.

\section{Robert Melton Leach, B.E.M.}

Mr. R. M. Leach, Experimental Officer in Parasitology at the Royal Army Medical College, died there on 19th January, 1967. Practical classes were memorable for his illustrations, many of which are preserved in the recently published "Atlas of Parasitology" by Jeffrey and Leach and in MacFarlane's "Short Synopsis of Human Protozoology and Helminthology." 